\title{
ROBERTSON'S CONJECTURE ON THE COEFFICIENTS OF CLOSE-TO-CONVEX FUNCTIONS
}

\author{
Y. LEUNG
}

Abstract. We use an inequality due to Lebedev and Milin to prove a conjecture made by M.S. Robertson on the coefficients of close-to-convex functions.

Let $S$ denote the class of functions

$$
f(z)=z+a_{2} z^{2}+\ldots
$$

that are regular and univalent in the unit disk $|z|<1$. Also, let $S^{*}$ denote the subclass of $S$ consisting of functions which map the unit disk onto domains which are starlike with respect to the origin. A function $f(z)$ in $S$ is close-to-convex if there exists a function $g(z)$ in $S^{*}$ such that

$$
\operatorname{Re} e^{i \alpha} \frac{z f^{\prime}(z)}{g(z)}>0
$$

for $|z|<1$, with $\alpha$ real.

In this note, we prove a conjecture raised by M. S. Robertson in [5], namely,

$$
|n| a_{n}|-m| a_{m}|| \leqslant\left|n^{2}-m^{2}\right|
$$

for all $f(z)=z+\sum_{n=2}^{\infty} a_{n} z^{n}$ that are close-to-convex.

ThEOREM. If $f(z)=z+\sum_{n=2}^{\infty} a_{n} z^{n}$ is close-to-convex, then

$$
|n| a_{n}|-m| a_{m}|| \leqslant\left|n^{2}-m^{2}\right|
$$

for all positive $n$ and $m$. Strict inequality holds for all $n$ and $m$, with $n \neq m$, unless $f(z)$ is a rotation of the Koebe function $z /(1-z)^{2}$.

COROLlaRY 1. For every close-to-convex function,

$$
\begin{gathered}
\left|a_{n}\right| \leqslant n, \quad n=2,3, \ldots, \\
-3+2 / n \leqslant\left|a_{n}\right|-\left|a_{n-1}\right| \leqslant 2-1 / n .
\end{gathered}
$$

The first part of the corollary is due to M. Reade [4].

The proof of the theorem depends on a lemma due to T. H. MacGregor [2], concerning the class $P$ of functions

Received by the editors June 19, 1978.

AMS (MOS) subject classifications (1970). Primary 30A32, 30A34.

Key words and phrases. Close-to-convex univalent functions, Lebedev-Milin inequality. 


$$
p(z)=1+\sum_{n=1}^{\infty} p_{n} z^{n}
$$

analytic and of positive real part in $|z|<1$.

LEMMA 1. Let $p \in P$, and let $\lambda_{n} \geqslant 0$. If $q(z)=\sum_{n=1}^{\infty} \lambda_{n} p_{n} z^{n}$ is analytic in $|z|<1$ and $\operatorname{Re} q(z) \leqslant M$ for some positive $M$, then $\sum_{n=1}^{\infty} \lambda_{n}\left|P_{n}\right|^{2} \leqslant 2 M$.

Corollary to THE LEMma. For each $p \in P$ and for each finite sequence $\left\{\lambda_{k}\right\}_{k=1}^{n}$ with $\lambda_{k} \geqslant 0$, there exists a $\zeta$ with $|\zeta|=1$ such that

$$
\sum_{k=1}^{n} \lambda_{k}\left|p_{k}-\zeta^{k}\right|^{2} \leqslant \sum_{k=1}^{n} \lambda_{k}
$$

Proof OF THE COROLlary. Apply the lemma to $q(z)=\sum_{k=1}^{n} \lambda_{k} p_{k} z^{k}$. This gives

$$
\begin{aligned}
\sum_{k=1}^{n} \lambda_{k}\left|p_{k}-\zeta^{k}\right|^{2} & =\sum_{k=1}^{n} \lambda_{k}\left|p_{k}\right|^{2}-2 \operatorname{Re} q(\bar{\zeta})+\sum_{k=1}^{n} \lambda_{k} \\
& \leqslant 2 M-2 \operatorname{Re} q(\bar{\zeta})+\sum_{k=1}^{n} \lambda_{k} .
\end{aligned}
$$

Choosing $\zeta$ with $|\zeta|=1$ so that $\operatorname{Re} q(\bar{\zeta})=M=\max _{|z|=1} \operatorname{Re}\{q(z)\}$, we obtain the result.

LEMMA 2. If $\sum_{k=0}^{\infty} D_{k} z^{k}=\exp \left(\sum_{k=1}^{\infty} A_{k} z^{k}\right)$, and both functions are analytic in $|z|<1$, then

$$
\sum_{k=0}^{n-1}\left|D_{k}\right|^{2} \leqslant n \exp \left\{\sum_{k=1}^{n}\left(k\left|A_{k}\right|^{2}-\frac{k^{2}\left|A_{k}\right|^{2}}{n}-\frac{1}{k}\right)+1\right\}
$$

with equality if and only if $A_{k}=c^{k} / k,|c|=1, k=1,2, \ldots, n-1$.

A proof of this so-called Lebedev-Milin inequality can be found in $D$. Aharonov's lecture notes [1, Theorem 1.2]. A similar proof can be found in Pommerenke's book [3, Lemma 3.3].

LEMMA 3. With the notations above, we have

$$
\begin{aligned}
\sum_{k=0}^{n-1}\left|D_{k}\right|^{2}+\frac{\left|D_{n}\right|^{2}}{2} \leqslant \frac{2 n+1}{2} \exp \left\{\sum_{k=1}^{n}\left(\frac{k^{2}}{n(2 n+1)}+k-\frac{k^{2}}{n}\right)\left|A_{k}\right|^{2}\right. \\
\left.-\sum_{k=1}^{n} \frac{1}{k}-\frac{1}{2 n+1}+1\right\},
\end{aligned}
$$

with equality if and only if $A_{k}=c^{k} / k,|c|=1, k=1,2, \ldots, n$. 
Proof. We imitate the proof as given in [1, Theorem 1.2]. Differentiating both sides of

$$
\sum_{k=0}^{\infty} D_{k} z^{k}=\exp \left(\sum_{k=1}^{\infty} A_{k} z^{k}\right)
$$

and comparing the coefficients of $z^{n-1}$, we get

$$
n D_{n}=\sum_{k=1}^{n} k A_{k} D_{n-k}
$$

By the Cauchy-Schwarz inequality,

$$
\left|D_{n}\right|^{2} \leqslant \sum_{k=1}^{n} \frac{k^{2}\left|A_{k}\right|^{2}}{n^{2}} \sum_{k=0}^{n-1}\left|D_{k}\right|^{2} .
$$

Therefore,

$$
\begin{aligned}
\sum_{k=0}^{n-1}\left|D_{k}\right|^{2}+\frac{\left|D_{n}\right|^{2}}{2} & \leqslant \sum_{k=0}^{n-1}\left|D_{k}\right|^{2}+\frac{1}{2} \sum_{k=1}^{n} \frac{k^{2}\left|A_{k}\right|^{2}}{n^{2}} \sum_{k=0}^{n-1}\left|D_{k}\right|^{2} \\
& =\left(1+\frac{1}{2} \sum_{k=1}^{n} \frac{k^{2}\left|A_{k}\right|^{2}}{n^{2}}\right) \sum_{k=0}^{n-1}\left|D_{k}\right|^{2} \\
& =\frac{2 n+1}{2 n}\left(1+\sum_{k=1}^{n} \frac{k^{2}\left|A_{k}\right|^{2}}{n(2 n+1)}-\frac{1}{2 n+1}\right) \sum_{k=0}^{n-1}\left|D_{k}\right|^{2} \\
& \leqslant \frac{2 n+1}{2 n} \exp \left\{\sum_{k=1}^{n} \frac{k^{2}\left|A_{k}\right|^{2}}{n(2 n+1)}-\frac{1}{2 n+1}\right\} \sum_{k=0}^{n-1}\left|D_{k}\right|^{2},
\end{aligned}
$$

where we applied the inequality $1+x \leqslant e^{x}$ with

$$
x=\sum_{k=1}^{n} \frac{k^{2}\left|A_{k}\right|^{2}}{n(2 n+1)}-\frac{1}{2 n+2} .
$$

Finally, applying Lemma 2 to estimate the last term of the above inequality, we get

$$
\begin{array}{r}
\sum_{k=0}^{n-1}\left|D_{k}\right|^{2}+\frac{\left|D_{n}\right|^{2}}{2} \leqslant \frac{2 n+1}{2} \exp \left\{\sum_{k=1}^{n}\left(\frac{k^{2}}{n(2 n+1)}+k-\frac{k^{2}}{n}\right)\left|A_{k}\right|^{2}\right. \\
\left.-\sum_{k=1}^{n} \frac{1}{k}-\frac{1}{2 n+1}+1\right\} .
\end{array}
$$

This completes the proof of the first part of the lemma.

The statement about the case of equality follows in the same way as in [1, Theorem 1.2], and we skip the proof. 
LEMMA 4. If $g(z)=z+\sum_{n=2}^{\infty} b_{n} z^{n}$ is in $S^{*}$, for each positive integer $n$, there exists $a \zeta$ with $|\zeta|=1$ such that

$$
1+\sum_{k=1}^{n-1}\left|b_{k+1}-\zeta b_{k}\right|^{2}+\frac{\left|b_{n+1}-\zeta b_{n}\right|^{2}}{2} \leqslant n+\frac{1}{2}
$$

Proof. If $g(z) \in S^{*}$, then it is well known that

$$
\frac{z g^{\prime}(z)}{g(z)}=p(z)=1+\sum_{n=1}^{\infty} p_{n} z^{n}
$$

with $p(z)$ in $P$. Hence

$$
\log \frac{g(z)}{z}=\sum_{n=1}^{\infty} \frac{p_{n}}{n} z^{n} .
$$

Take a $\zeta$ on the unit circle, then

$$
\begin{aligned}
(1-\zeta z) \frac{g(z)}{z} & =1+\sum_{k=1}^{\infty}\left(b_{k+1}-\zeta b_{k}\right) z^{k} \\
& =\sum_{k=0}^{\infty} D_{k} z^{k}, \text { say. }
\end{aligned}
$$

On the other hand,

$$
\begin{aligned}
\log (1-\zeta z) \frac{g(z)}{z} & =\sum_{k=1}^{\infty} \frac{\left(p_{k}-\zeta^{k}\right)}{k} z^{k} \\
& =\sum_{k=1}^{\infty} A_{k} z^{k}, \text { say. }
\end{aligned}
$$

Then,

$$
\sum_{k=0}^{\infty} D_{k} z^{k}=\exp \left(\sum_{k=0}^{\infty} A_{k} z^{k}\right)
$$

By Lemma 3,

$$
\begin{array}{r}
\sum_{k=0}^{n-1}\left|D_{k}\right|^{2}+\frac{\left|D_{n}\right|^{2}}{2} \leqslant \frac{2 n+1}{2} \exp \left\{\sum_{k=1}^{n}\left(\frac{k^{2}}{n(2 n+1)}+k-\frac{k^{2}}{n}\right)\left|A_{k}\right|^{2}\right. \\
\left.\quad-\sum_{k=1}^{n} \frac{1}{k}-\frac{1}{2 n+1}+1\right\} \\
=\frac{2 n+1}{2} \exp \left\{\sum_{k=1}^{n}\left(\frac{k^{2}}{n(2 n+1)}+k-\frac{k^{2}}{n}\right) \frac{\left|p_{k}-\zeta^{k}\right|^{2}}{k^{2}}\right. \\
\left.-\sum_{k=1}^{n} \frac{1}{k}-\frac{1}{2 n+1}+1\right\}
\end{array}
$$


By the corollary to Lemma 1 , we can pick a $\zeta$ with $|\zeta|=1$ such that

$$
\begin{aligned}
& \sum_{k=1}^{n}\left(\frac{k^{2}}{n(2 n+1)}+k-\frac{k^{2}}{n}\right) \frac{\left|p_{k}-\zeta^{k}\right|^{2}}{k^{2}} \\
& \leqslant \sum_{k=1}^{n}\left(\frac{k^{2}}{n(2 n+1)}+k-\frac{k^{2}}{n}\right) \frac{1}{k^{2}} \\
& =\sum_{k=1}^{n}\left(\frac{1}{n(2 n+1)}+\frac{1}{k}-\frac{1}{n}\right) \\
& =\frac{1}{2 n+1}+\sum_{k=1}^{n} \frac{1}{k}-1 .
\end{aligned}
$$

Hence, there exists a $\zeta$ with $|\zeta|=1$ to make the exponent in (*) nonpositive, and we have

$$
\sum_{k=0}^{n-1}\left|D_{k}\right|^{2}+\frac{\left|D_{n}\right|^{2}}{2} \leqslant \frac{2 n+1}{2}=n+\frac{1}{2} .
$$

This is the statement of Lemma 4.

PROOF OF THE THEOREM. If $f(z)$ is close-to-convex, then $e^{i \alpha} z f^{\prime}(z)=$ $r(z) g(z)$, where $\operatorname{Re} r(z)>0$ and

$$
r(z)=e^{i \alpha}+\sum_{k=1}^{\infty} r_{k} z^{k} .
$$

For $|\zeta|=1$, we have

$$
e^{i \alpha}(1-\zeta z) f^{\prime}(z)=(1-\zeta z) \frac{g(z)}{z} r(z)
$$

or,

$$
\begin{aligned}
e^{i \alpha}\left\{1+\sum_{n=1}^{\infty}[\right. & {\left.\left[(n+1) a_{n+1}-\zeta n a_{n}\right] z^{n}\right\} } \\
= & {\left[1+\sum_{k=1}^{\infty}\left(b_{k+1}-\zeta b_{k}\right) z^{k}\right]\left[e^{i \alpha}+\sum_{k=1}^{\infty} r_{k} z^{k}\right] }
\end{aligned}
$$

Comparing the coefficients of $z^{n}$ on both sides, we get

$$
\begin{aligned}
e^{i \alpha}\left[(n+1) a_{n+1}-\zeta n a_{n}\right] & =r_{n}+r_{n-1}\left(b_{2}-\zeta b_{1}\right)+\ldots+e^{i \alpha}\left(b_{n+1}-\zeta b_{n}\right) \\
& =\sum_{k=0}^{n} r_{n-k} D_{k},
\end{aligned}
$$

where $r_{0}=e^{i \alpha}$ and $D_{k}=b_{k+1}-\zeta b_{k}$. To the first $n$ terms of the right-hand side, we apply the inequality $|a b| \leqslant|a / 2|^{2}+|b|^{2}$, and to the last term the inequality $|b| \leqslant \frac{1}{2}+|b|^{2} / 2$. We obtain, 


$$
\begin{aligned}
\left|(n+1) a_{n+1}-\zeta n a_{n}\right| \leqslant & \left|\frac{r_{n}}{2}\right|^{2}+1+\left|\frac{r_{n-1}}{2}\right|^{2} \\
& +\left|b_{2}-b_{1}\right|^{2}+\ldots+\frac{1}{2}+\frac{1}{2}\left|b_{n+1}-\zeta b_{n}\right|^{2} \\
& \leqslant n+\frac{1}{2}+1+\sum_{k=1}^{n-1}\left|b_{k+1}-b_{k}\right|^{2}+\frac{1}{2}\left|b_{n+1}-\zeta b_{n}\right|^{2}
\end{aligned}
$$

where we applied the well-known estimate $\left|r_{k}\right| \leqslant 2$, for $k=1,2, \ldots$ By Lemma 4,

$$
\left|(n+1) a_{n+1}-\zeta n a_{n}\right| \leqslant n+\frac{1}{2}+n+\frac{1}{2}=2 n+1,
$$

for some $\zeta$ with $|\zeta|=1$. But by the triangle inequality,

$$
|(n+1)| a_{n+1}|-n| a_{n}|| \leqslant\left|(n+1) a_{n+1}-\zeta n a_{n}\right| .
$$

Hence,

$$
|(n+1)| a_{n+1}|-n| a_{n}|| \leqslant 2 n+1 .
$$

The general case of inequality follows from this by induction.

In the case of equality, a close inspection of the proof shows that $r(z)$ has the form $(1+t z) /(1-t z)$, with $|t|=1$. Hence, we have $z f^{\prime}(z)=((1+$ $t z) /(1-t z)) g(z)$. Multiplying both sides by $(1-t z)$, we get

$$
(1-t z) \sum_{n=1}^{\infty} n a_{n} z^{n}=(1+t z) \sum_{n=1}^{\infty} b_{n} z^{n} .
$$

Comparing the coefficient of $z^{n+1}$ on both sides, we obtain

$$
(n+1) a_{n+1}-t n a_{n}=b_{n+1}+t b_{n} \text {. }
$$

Hence,

$$
2 n+1=|(n+1)| a_{n+1}|-n| a_{n}|| \leqslant\left|b_{n+1}+t b_{n}\right| .
$$

So our starlike function $g(z)$ must be a rotation of the Koebe function and has the form $z /(1-t z)^{2}$; hence $f(z)$ is $z /(1-t z)^{2}$ also.

\section{REFERENCES}

1. D. Aharonov, Special topics in univalent functions, Lecture notes, Univ. Maryland, 1971.

2. T. H. MacGregor, An inequality concerning analytic functions with a positive real part, Canad. J. Math. 21 (1969), 1172-1177.

3. Christian Pommerenke, Univalent functions, Vandenhoeck \& Ruprecht, Göttingen, 1975.

4. M. O. Reade, On close-to-convex univalent function, Michigan Math. J. 3 (1955), 59-62.

5. M. S. Robertson, Quasi-subordination and coefficient conjectures, Bull. Amer. Math. Soc. 76 (1970), 1-9.

Department of Mathematics, University of Michigan, ANN ARbor, Michigan 48109 Current address: On Hing Company, 137 Temple Street, Hong Kong 